\title{
Universal trapping in a three-beam optical lattice
}

\author{
T. V. Raziman and Olivier J. F. Martin* \\ Nanophotonics and Metrology Laboratory, Swiss Federal Institute of Technology Lausanne, 1015 Lausanne, Switzerland
}

(Received 4 July 2018; published 22 August 2018)

\begin{abstract}
We study electromagnetic trapping in an optical lattice formed by three equiangular in-plane beams. We demonstrate analytically that this optical lattice offers stable trapping sites for particles satisfying specific symmetries, irrespective of the exact nature of their electromagnetic response. Under small displacements, the particles are shown to be subject to equal restoring forces along all directions and the trap is isotropic. Though the intensity distribution of the trap forms a perfect hexagonal lattice, differences in phase variation along opposite directions cause the restoring force to be asymmetric for large displacements, resulting in a force landscape possessing only threefold symmetry. We then show numerically that this asymmetry affects the optical binding force between particles in adjacent trap positions and results in unequal shifts of their equilibrium positions. Universal trapping in the optical lattice combined with this asymmetric mechanical response of trapped particle pairs promises rich optomechanical effects.
\end{abstract}

DOI: 10.1103/PhysRevA.98.023420

\section{INTRODUCTION}

Electromagnetic fields can transfer linear and angular momenta to particles, resulting in forces and torques on them [1]. Both propagating waves and field intensity gradients have been used to generate optical forces and torques [2-4]. Optical tweezers utilize forces arising from strong field gradients to trap nanoparticles at desired locations, granting an unprecedented ability to isolate, study, and modify them [5]. Trapping has been performed on a wide range of objects from atoms to particles and cells [6]. The field of optical manipulation has consistently gained attention, especially since the advent of plasmonic trapping, and has resulted in a multitude of tested as well as promising technological applications in nanotechnology, biology, and optics [7-21].

Optical tweezer arrays permit the trapping of multiple particles simultaneously and are fundamental to achieving large-scale optical nanomanipulation [22,23]. Many techniques have been introduced to generate such arrays including structured light fields [24,25], plasmonic arrays [17,19], beam shaping using holograms [26-30], and micromirror arrays [31]. Standing-wave patterns formed by interfering beams can be used to generate periodic arrays of trapping sites [32-35]. These optical lattices have the ability to trap multiple particles in one-, two-, and three-dimensional arrays, creating optical matter [36-38]. When multiple particles are trapped in an electromagnetic field, the interaction between them gives rise to interparticle forces which strongly modify the behavior of the trapping forces [39-41]. This can result in optical binding between particles $[42,43]$ and can be used for the self-assembly of crystals of the particles [44-50].

In this paper, we study trapping in a hexagonal optical lattice formed by three equiangular beams in a plane. Rather than constraining ourselves to specific geometries or approximations,

*olivier.martin@epfl.ch we provide a general analytical treatment that shows that the three-beam trap admits a stable equilibrium for any particle, irrespective of the exact nature of its electromagnetic response, as long as it satisfies specific symmetries. We also demonstrate that though the electric field intensity landscape in the lattice is hexagonally symmetric, phase variations result in a flip asymmetry in forces. To obtain a better physical understanding of the general theoretical results, we also compare them with the dipole approximation and numerical calculations using the surface integral equation (SIE) method [51-53]. Finally, we show that the force asymmetry further manifests in the optical binding between particles trapped in the lattice.

\section{NUMERICAL RESULTS}

The optical force $\mathbf{F}$ on a particle can be computed using the formula [1]

$$
F_{i}=\oint_{S}\left\langle\sigma_{i j}\right\rangle n_{j} d S
$$

where $S$ is a surface surrounding the particle with normal vector $\mathbf{n}$ and $\left\langle\sigma_{i j}\right\rangle$ is the time-averaged Maxwell stress tensor. For monochromatic fields with the time dependence $\exp (-i \omega t)$, the time-averaged stress tensor can be expressed in terms of the complex electric field $\mathbf{E}$ and magnetic field $\mathbf{H}$ as

$$
\begin{aligned}
\left\langle\sigma_{i j}\right\rangle= & \frac{1}{2} \operatorname{Re}\left[\epsilon_{0} E_{i}^{*} E_{j}+\mu_{0} H_{i}^{*} H_{j}\right. \\
& \left.-\frac{1}{2} \delta_{i j} \sum_{k}\left(\epsilon_{0} E_{k}^{*} E_{k}+\mu_{0} H_{k}^{*} H_{k}\right)\right] .
\end{aligned}
$$

Here the electric and magnetic fields include both the incident field and the scattered field from the particle. In addition, $\epsilon_{0}$ and $\mu_{0}$ are the electric permittivity and magnetic permeability of the background medium assumed to be a vacuum and $\delta_{i j}$ is the Kronecker delta. 

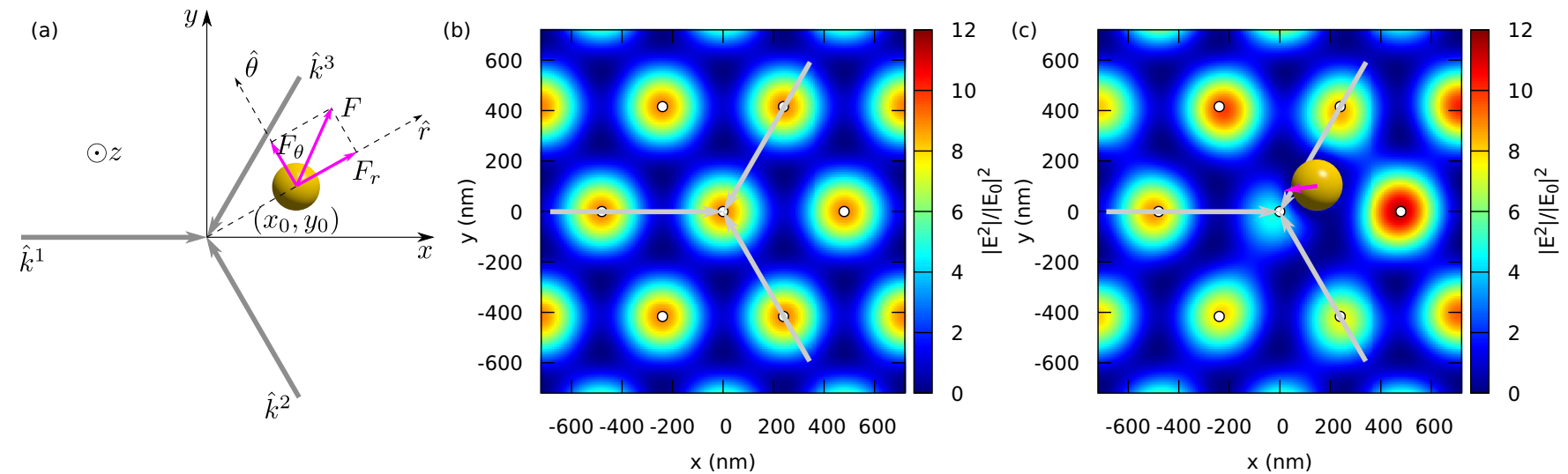

FIG. 1. (a) Schematic of the system. Three coherent plane waves with $\lambda=720 \mathrm{~nm}$ and electric field along $z$ with electric field intensity $\left|E_{0}\right|^{2}$ are incident along directions $\hat{k}^{1}=\hat{x}, \hat{k}^{2}=-\frac{1}{2} \hat{x}+\frac{\sqrt{3}}{2} \hat{y}$, and $\hat{k}^{3}=-\frac{1}{2} \hat{x}-\frac{\sqrt{3}}{2} \hat{y}$. A gold sphere of 100-nm radius is placed with its center at $\mathbf{r}_{0}=\left(x_{0}, y_{0}\right)$. (b) In the absence of the sphere, the beams form a hexagonal electric field intensity landscape. (c) Placing the sphere disturbs this landscape, resulting in a force on the sphere (magenta arrow). Locations of the seven intensity maxima closest to the origin when the sphere is absent are marked with white circles in (b) and (c).

Before we delve into the general theory, let us look at a concrete example. A spherical gold particle of radius $r=$ $100 \mathrm{~nm}$ located on the $x y$ plane with its center at $\left(x_{0}, y_{0}\right)$ is illuminated by three plane waves with wavelength $\lambda=720 \mathrm{~nm}$ having electric field polarized along $z$ and propagating in plane at an angle of $120^{\circ}$ from each other as shown in Fig. 1(a). The propagation vectors of the three waves are along directions $\hat{k}^{1}=\hat{x}, \hat{k}^{2}=-\frac{1}{2} \hat{x}+\frac{\sqrt{3}}{2} \hat{y}$, and $\hat{k}^{3}=-\frac{1}{2} \hat{x}-\frac{\sqrt{3}}{2} \hat{y}$. Although such in-plane illumination is unrealistic in the context of trapping experiments, we are interested only in in-plane forces and neglect the forward scattering force, making the approximation valid. The three plane waves have equal intensities and are coherent, and their phases are chosen such that all three beams are in phase at the origin. Under these conditions, in the absence of the sphere, the electric field intensity forms a perfect hexagonal lattice with a lattice constant of $\frac{2 \lambda}{3}=480 \mathrm{~nm}$, showing a variation from zero to nine times the incident intensity of a single beam as seen in Fig. 1(b).

Placing the sphere changes the field profile of the optical lattice. The near-field modification due to the sphere (with the permittivity of gold described by a Drude fit to experimental data [54,55]) located at $(150 \mathrm{~nm}, 100 \mathrm{~nm})$ is computed using the SIE and plotted in Fig. 1(c). Comparing with Fig. 1(b), the field intensity at the origin is seen to be considerably weakened whereas the intensities at other locations such as $\left(\frac{2 \lambda}{3}, 0\right)$ are enhanced. The near-field landscape modification results in an optical force $\mathbf{F}$ on the sphere according to Eqs. (1) and (2). The force can be decomposed into a radial component $F_{r}$ and a tangential component $F_{\theta}$. The former moves the sphere away from the origin (when positive) or towards it (when negative). A negative radial force is thus a restoring force, resulting in stable equilibrium.

The radial and tangential components of the optical force on the particle under displacement in the $x y$ plane are shown in Fig. 2. Each point in the graph corresponds to an SIE force calculation similar to Fig. 1(c) for the sphere centered at $\left(x_{0}, y_{0}\right)$. The radial component of the force is negative in a region centered at the origin. The particle has a stable equilibrium there and returns to the origin if displaced slightly. The tangential forces are very weak in comparison: For a displacement under $100 \mathrm{~nm}$, the maximum value of the radial force is more than five times that of the tangential force. What seems surprising at first glance is that the force in Fig. 2 does not have the sixfold symmetry of the hexagonal lattice in Fig. 1(b). Instead, it possesses a threefold symmetry corresponding to the three illumination beams. In particular, the restoring force is
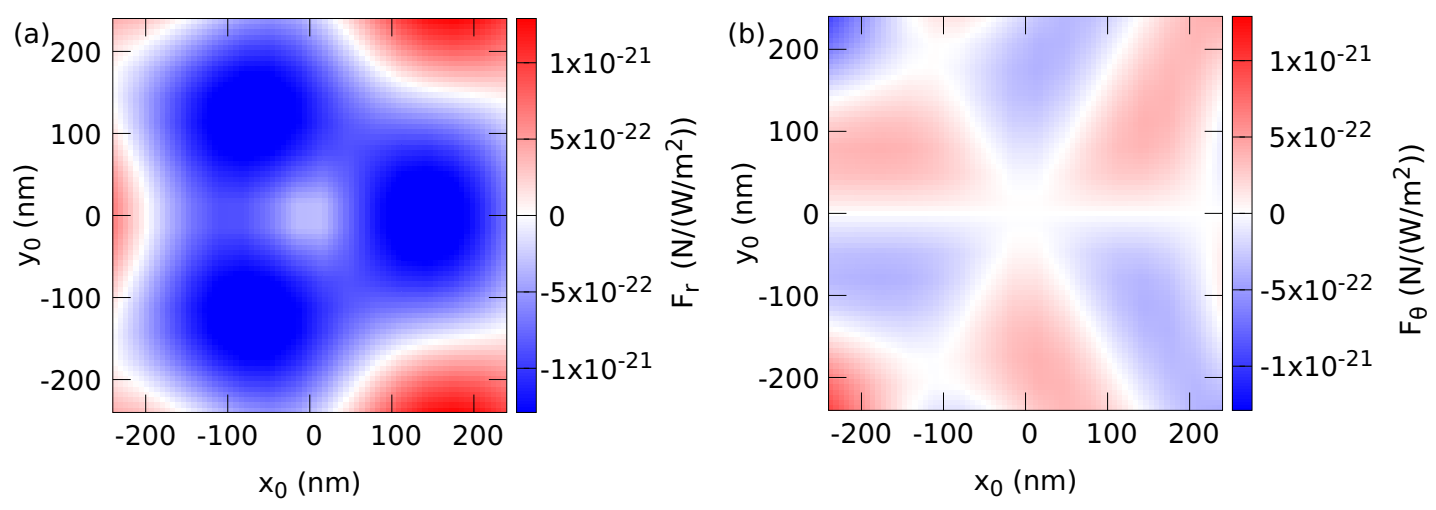

FIG. 2. Optical forces on the sphere as a function of the position of its center in the $x y$ plane: (a) radial component $F_{r}$ and (b) tangential component $F_{\theta}$. 
asymmetric for positive and negative displacements about the $x$ axis. The particle is more stable for displacements along $+x$ than along $-x$, although the initial field pattern is perfectly symmetric along that direction.

The reason for this asymmetry is that the force arises not merely from the intensity gradient but depends on the phase as well. Though the different peaks in the lattice have the same intensity, they achieve their maxima at different times, as seen in Supplemental Material [56], and hence have a phase difference. We will now explain the forces observed by means of an analytical treatment of the system.

\section{THEORY}

Using the properties of the stress tensor in Eq. (2) and symmetry, the optical force on an arbitrary object with threefold rotational symmetry about the $z$ axis and mirror symmetry about the $x y$ plane and the $x z$ plane is derived in the Appendix. When the object is centered at $\mathbf{r}_{0}$, the force is given by

$$
\begin{aligned}
\mathbf{F}\left(\mathbf{r}_{0}\right)= & \left\{\left[\cos \phi^{21} R^{2}+\cos \phi^{32}+\cos \phi^{13} R\right]\left(U^{23} \hat{x}\right)\right. \\
& \left.+\left[\sin \phi^{21} R^{2}+\sin \phi^{32}+\sin \phi^{13} R\right]\left(V^{23} \hat{y}\right)\right\},
\end{aligned}
$$

where $\phi^{m n}$ is the relative phase between the $m$ th and $n$th beams at $\mathbf{r}_{0}, R$ is the $120^{\circ}$ rotation operator, and $U^{23}$ and $V^{23}$ are real parameters depending on the electromagnetic response of the object. Thus the force response of the particle in the entire three-beam landscape is dictated only by the two real parameters $U^{23}$ and $V^{23}$ defined in the Appendix.

\section{A. Stability of the trap}

Let us now perform the stability analysis of the trap. Suppose that the particle is only slightly displaced from $(0,0)$ to $\mathbf{r}_{0}=\left(r_{0} \cos \theta, r_{0} \sin \theta\right)$. As a result, the phase terms in Eq. (3) become

$$
\begin{aligned}
\phi^{21} & =\frac{\pi r_{0}}{\lambda}(-3 \cos \theta+\sqrt{3} \sin \theta), \\
\phi^{32} & =\frac{\pi r_{0}}{\lambda}(-2 \sqrt{3} \sin \theta), \\
\phi^{13} & =\frac{\pi r_{0}}{\lambda}(3 \cos \theta+\sqrt{3} \sin \theta) .
\end{aligned}
$$

To study the small displacement limit of $r_{0} / \lambda \rightarrow 0$, we substitute the expression for the phase terms in Eq. (3) and perform a Taylor expansion retaining only terms up to $O\left(r_{0} / \lambda\right)$. The cosine terms cancel because $1+R+R^{2}=0$ [see Eq. (A5b)] and we are left with

$$
\begin{aligned}
\mathbf{F}\left(\mathbf{r}_{0}\right) & \approx \frac{\pi r_{0}}{\lambda}\left[3 \cos \theta\left(R-R^{2}\right)-3 \sqrt{3} \sin \theta\right]\left(V^{23} \hat{y}\right) \\
& =\frac{3 \sqrt{3} \pi V^{23}}{\lambda}\left(-\mathbf{r}_{0}\right) .
\end{aligned}
$$

That is, up to the leading order, the force is radial and of a restoring nature when $V^{23}>0$. We thus have a stable harmonic trap with equal restoring force for small displacements along all directions.

However, we had seen in Fig. 2 that the restoring force is not cylindrically symmetric. In particular, the force is not equal for positive and negative displacements along the $x$ axis. Let us now look at the source of this asymmetry by dropping the small displacement approximation and limiting ourselves to the $x$ axis. If the particle is positioned such that $\mathbf{r}_{0}=\left(x_{0}, 0\right)$, we have $\phi^{21}=-\frac{3 \pi x_{0}}{\lambda}=-\phi^{13}$. Hence the expression for force in Eq. (3) becomes

$$
\begin{aligned}
\mathbf{F}\left(x_{0}\right)= & {\left[1+\cos \phi^{21}\left(R^{2}+R\right)\right]\left(U^{23} \hat{x}\right) } \\
& +\sin \phi^{21}\left[R^{2}-R\right]\left(V^{23} \hat{y}\right) \\
= & {\left[1-\cos \left(\frac{3 \pi x_{0}}{\lambda}\right)\right] U^{23} \hat{x}-\sqrt{3} \sin \left(\frac{3 \pi x_{0}}{\lambda}\right) V^{23} \hat{x} . }
\end{aligned}
$$

As expected, the force is along $x$ and vanishes at the origin. The behavior when $x_{0} / \lambda \rightarrow 0$ agrees with the result in Eq. (5). For small values of $x_{0}$, it is the latter term that dominates and hence the particle experiences a stable harmonic potential if $V^{23}>0$. However, as the displacement becomes significant, the $U^{23}$ term makes the potential asymmetric and is the reason why the particle experiences different forces if displaced to the left and right. In Fig. 3, the restoring force on the particle is plotted for displacements along $x$ with the fit to Eq. (6). The agreement is seen to be perfect. The valleys are deeper than the peaks, explaining why displacement along $+x$ is more stable than along $-x$ (note the position of the origin in Fig. 3).

Though the particle has stable equilibrium at the origin only when $V^{23}>0$, there exist other locations for $V^{23}<0$ where the radial force is stabilizing. To see this, we place the particle at an intensity minimum of the lattice at $\mathbf{r}_{\mathbf{m}}=\left(\frac{2 \lambda}{3}, \frac{2 \lambda}{3 \sqrt{3}}\right)$ and displace it slightly to $\mathbf{r}_{\mathbf{m}}+\mathbf{r}_{0}$. Following similar steps as in Eqs. (4) and (5), the optical force in this situation is found to be

$$
\begin{aligned}
\mathbf{F}\left(\mathbf{r}_{\mathbf{m}}+\mathbf{r}_{0}\right)= & \left\{-\sin \left(\frac{2 \pi}{3}\right)\left[\sin \phi^{21} R^{2}+\sin \phi^{32}\right.\right. \\
& \left.+\sin \phi^{13} R\right]\left(U^{23} \hat{x}\right)+\cos \left(\frac{2 \pi}{3}\right)\left[\sin \phi^{21} R^{2}\right. \\
& \left.\left.+\sin \phi^{32}+\sin \phi^{13} R\right]\left(V^{23} \hat{y}\right)\right\} \\
\approx & -\frac{9 U^{23}}{4 \lambda} r_{0} \hat{\theta}+\frac{3 \sqrt{3} \pi V^{23}}{2 \lambda} \mathbf{r}_{0}
\end{aligned}
$$

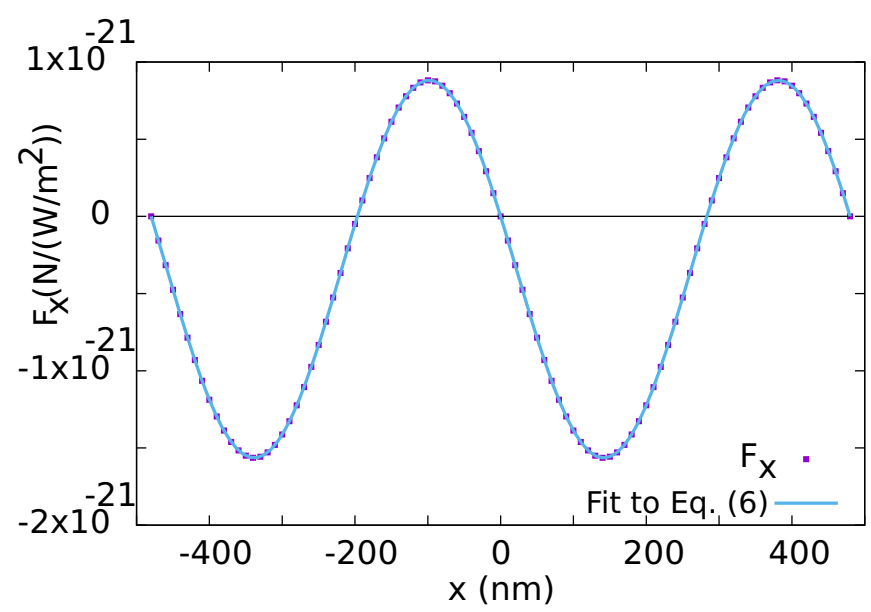

FIG. 3. Restoring force $F_{x}$ on the sphere for displacements along the $x$ axis. The SIE computation results (points) and the best-fit curve from Eq. (6) are shown. 
where the second step makes the small displacement approximation $r_{0} / \lambda \rightarrow 0$. The first term is a tangential force that does not affect the stability of the trap. However, its presence indicates that the optical force is nonconservative since its line integral on a circle around $\mathbf{r}_{m}$ is nonzero. The second term is a stabilizing radial force pushing the particle towards $\mathbf{r}_{m}$ for $V^{23}<0$, making intensity minima stable trap sites when $V^{23}<0$. Combining with the previous result that intensity maxima are stable trap sites for $V^{23}>0$, we have the surprising result that all spherical particles experience a stabilizing restoring force at some location in the three-beam optical trap irrespective of the underlying electromagnetic response which dictates the sign of $V^{23}$. Note that we have not used any electromagnetic properties of the particle other than linearity and any geometric properties other than symmetry (the particle should possess threefold rotational symmetry about the $z$ axis and mirror symmetries about the $x y$ plane and the $x z$ plane). Hence this treatment is valid for particles of all sizes and made out of any material and for other symmetrical shapes such as a cylindrical disk and an equilateral triangle. The three-beam optical lattice is thus a universal trap for all kinds of particles as long as they satisfy the requisite symmetry.

The only exception to this treatment is the case when $V^{23}$ becomes exactly zero during a sign flip. When this happens, according to Eq. (6), the force graph becomes tangent to the $x$ axis. That is, though the force vanishes, it is not a position of stable equilibrium but of neutral equilibrium. Similarly, when displaced tangentially from $\mathbf{r}_{m}$ in Eq. (7), the radial force vanishes but the tangential force is sufficient to take the particle away because of the lack of a restoring force. However, this case can usually be neglected since $V^{23}=0$ happens for a particle only for a specific wavelength and even small deviations away from it will take us back to one of the two cases of equilibria dealt with previously.

\section{B. Dipole approximation}

To understand the physical significance of the $U^{23}$ and $V^{23}$ parameters, let us consider the limit when the sphere is small enough to be treated as a dipole with dipole moment

$$
\mathbf{p}\left(\mathbf{r}_{0}\right)=\alpha \mathbf{E}_{0}\left(\mathbf{r}_{0}\right),
$$

where $\alpha$ is the complex polarizability of the sphere and $\mathbf{E}_{0}$ is the incident electric field at the location of the sphere $\mathbf{r}_{0}$. Under the dipole approximation, the force is given by [57]

$$
\mathbf{F}\left(\mathbf{r}_{0}\right)=\frac{1}{2} \operatorname{Re}\left(\left(\mathbf{p}^{*} \cdot \nabla\right) \mathbf{E}_{0}+\mu_{0} \frac{d \mathbf{p}}{d t} \times \mathbf{H}_{0}^{*}\right) .
$$

The first term arises from the electric part of the Lorentz force. However, since the dipole moment is always along the direction of the incident electric field $(z)$ and the electric field gradients are in the $x y$ plane, this term vanishes. Hence the only nonzero term is the second one, which results from the magnetic part of the Lorentz force, and can be written as

$$
\mathbf{F}\left(\mathbf{r}_{0}\right)=\frac{\omega \mu_{0}}{2} \operatorname{Im}\left(\alpha \mathbf{E}_{0} \times \mathbf{H}_{0}^{*}\right) .
$$

In the same fashion as before, we can express the total incident field as the superposition of the incident fields from the three beams,

$$
\begin{aligned}
\mathbf{F}\left(\mathbf{r}_{0}\right) & =\frac{\omega \mu_{0}}{2} \operatorname{Im} \sum_{m, n} \alpha \mathbf{E}_{0}^{m} \times \mathbf{H}_{0}^{n *} \\
& =\frac{\pi}{\lambda} \operatorname{Im} \sum_{m, n} \alpha\left(\mathbf{E}_{0}^{m} \cdot \mathbf{E}_{0}^{n *}\right) \hat{k}^{n} \\
& =\frac{\pi\left|E_{0}\right|^{2}}{\lambda} \operatorname{Im} \sum_{m, n} \alpha \exp \left(i \phi^{m n}\right) \hat{k}^{n} .
\end{aligned}
$$

Maxwell's equations have been used for the simplification. This expression can now be processed in the same manner as was done with Eq. (A6). For displacements along $x$, we find that

$$
\begin{aligned}
\mathbf{F}\left(x_{0}\right)= & -\left[1-\cos \left(\frac{3 \pi x_{0}}{\lambda}\right)\right] \operatorname{Im}(\alpha) \frac{\pi\left|E_{0}\right|^{2}}{\lambda} \hat{x} \\
& -3 \sin \left(\frac{3 \pi x_{0}}{\lambda}\right) \operatorname{Re}(\alpha) \frac{\pi\left|E_{0}\right|^{2}}{\lambda} \hat{x} .
\end{aligned}
$$

Comparing with Eq. (6), we obtain that

$$
\begin{aligned}
U^{23} & =-\operatorname{Im}(\alpha) \frac{\pi|E|_{0}^{2}}{\lambda}, \\
V^{23} & =\operatorname{Re}(\alpha) \frac{\sqrt{3} \pi|E|_{0}^{2}}{\lambda},
\end{aligned}
$$

under the dipole approximation. The $V^{23}$ term depends upon the real part of the polarizability. This suggests that this force term arises from the electric field intensity gradient. On the other hand, the $U^{23}$ term depends on the imaginary part of the polarizability and is thus related to the extinction. Since the real part of the polarizability of the sphere can change sign across a plasmon resonance, this suggests that $V^{23}$ will flip sign as well.

Numerically retrieved values of $U^{23}$ and $V^{23}$ from SIE simulations using Eq. (6) are plotted in Fig. 4 with solid lines. For a sphere of radius $r=50 \mathrm{~nm}, V^{23}$ is positive and dominates $U^{23}$ at high wavelengths [Fig. 4(a)]. This should not be unexpected since the polarizability of the sphere is dominantly real and positive at frequencies much lower than the resonance. As the wavelength is decreased, we approach resonance and the absolute value of $U^{23}$ is maximized. Further, $V^{23}$ passes through zero and changes sign across the resonance, resulting in a reversal in the direction of force [58]. The negative sign in $U^{23}$ can be found to be consistent on comparing Eqs. (6) and (12). We also computed the polarizability from the dipole moment induced on the sphere and evaluated $U^{23}$ and $V^{23}$ using Eq. (13), which are presented as dashed lines in Fig. 4(a). The agreement is nearly perfect, suggesting that modeling the sphere as a dipole is an excellent approximation in this limit.

When the sphere size is increased, the higher-order modes dominate and the dipole approximation is expected to fail. In Fig. 4(b) we keep the wavelength fixed at $600 \mathrm{~nm}$ and plot $U^{23}$ and $V^{23}$ as a function of the sphere radius. The deviation from the dipole approximation starts becoming noticeable below $100 \mathrm{~nm}$. The higher-order modes in the sphere cause reversals in the sign of both $U^{23}$ and $V^{23}$ for a radius around $160 \mathrm{~nm}$. 

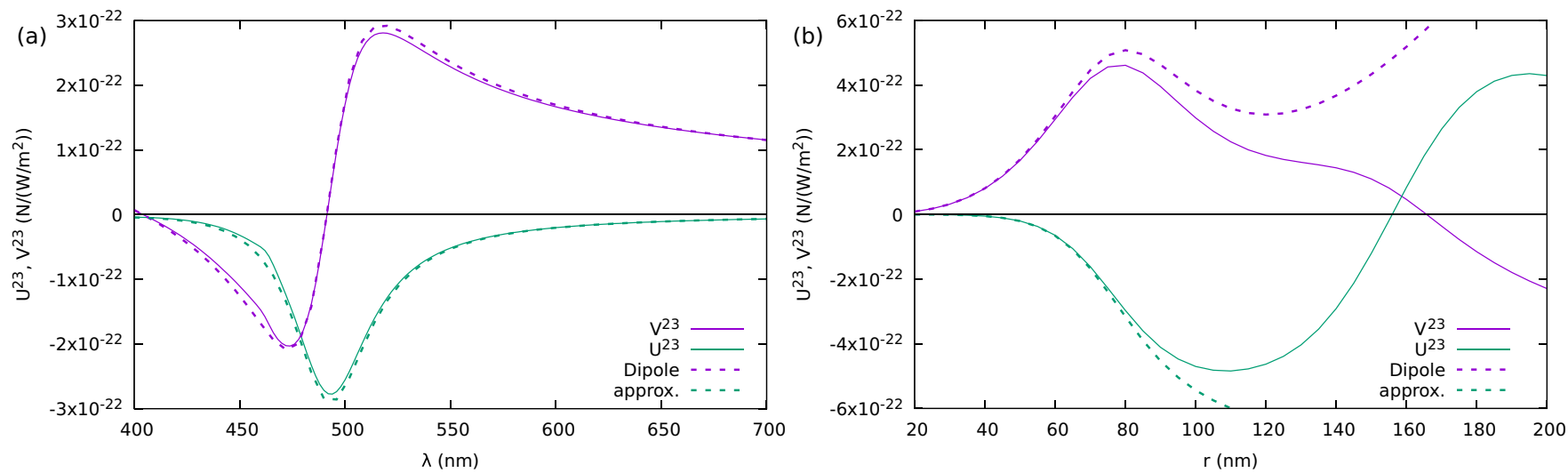

FIG. 4. Dependence of parameters $U^{23}$ and $V^{23}$ in Eq. (3) on (a) wavelength for fixed radius $r=50 \mathrm{~nm}$ and (b) radius for fixed wavelength $\lambda=600 \mathrm{~nm}$. Solid lines denote the values computed directly from the force in SIE simulations using Eq. (6), whereas dashed lines are calculated from the dipole moment according to Eq. (13).

A reversal in the sign of $U^{23}$ is impossible under the dipole approximation because that would lead to a negative imaginary part of the dipole moment and thus to negative extinction.

\section{OPTICAL BINDING FORCES}

The asymmetry in the force seen here could also manifest in the coupling between particles at different trap sites and thus the optical binding [59]. To study this, we consider the same system presented in Sec. II, with an additional identical gold particle. The two particles are placed at the two trap locations $(0,0)$ and $(480,0)$ and we compute the restoring force on each of them when they are displaced along the $x$ axis to locations $\left(x_{1}, 0\right)$ and $\left(x_{2}, 0\right)$, respectively. The geometry of the system is shown in Fig. 5(a). The electric field intensity for symmetric displacement by $100 \mathrm{~nm}\left(x_{1}=100 \mathrm{~nm}, x_{2}=380 \mathrm{~nm}\right)$ plotted in Fig. 5(b) clearly demonstrates field asymmetry.

The restoring force on the first particle is shown in Fig. 5(c). Keeping the second particle fixed at $(480 \mathrm{~nm}, 0)$ moves the equilibrium position of the first particle to the right by more than $100 \mathrm{~nm}$. Displacing the second particle has a tremendous effect on the equilibrium position of the first; moving it away by less than $50 \mathrm{~nm}$ brings the first particle back to the origin. On the other hand, the restoring force on the second particle in Fig. 5(d) shows a much weaker shift. When the first particle is kept fixed at the origin, the second particle is (a)

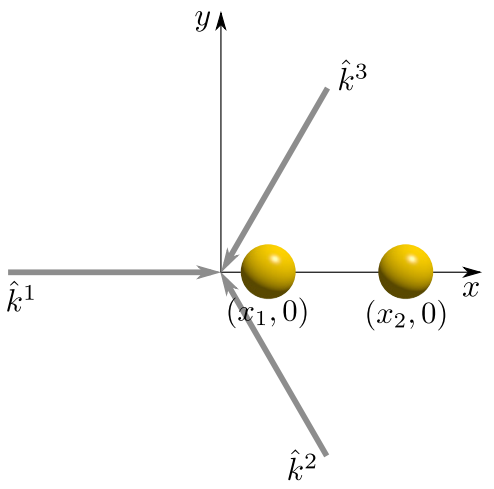

(c)

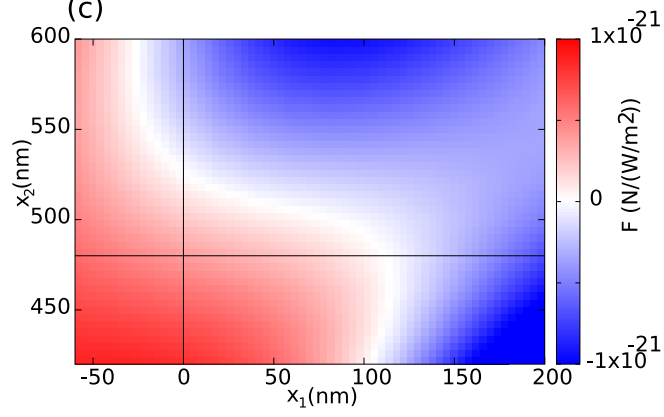

(b) 600
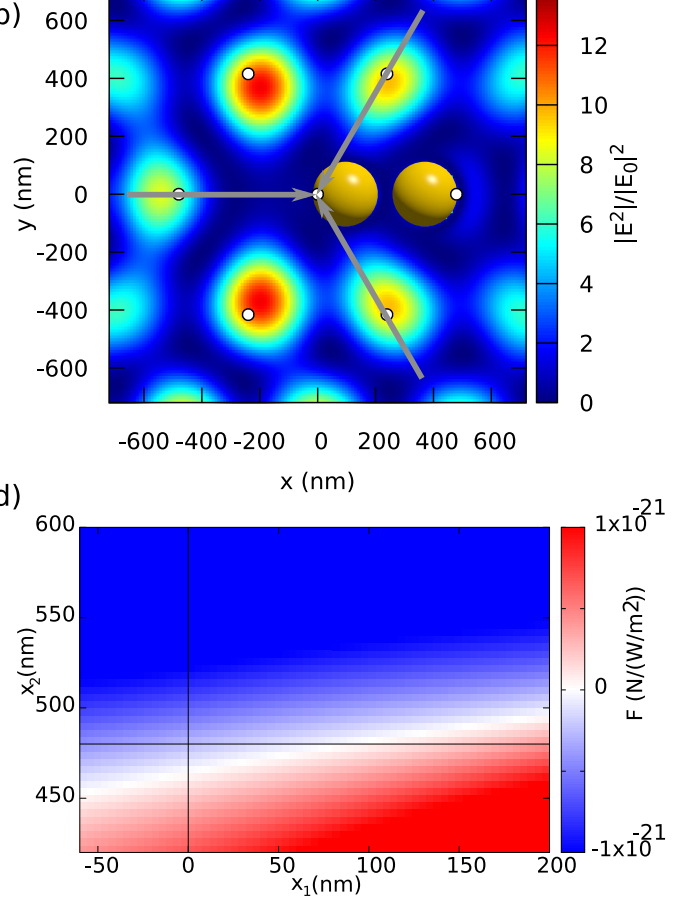

FIG. 5. (a) Schematic of the two-particle system. Two gold particles of 100-nm radius are placed at adjacent electric field intensity maxima at $(0,0)$ and $(480 \mathrm{~nm}, 0)$ and displaced along the $x$ axis to $\left(x_{1}, 0\right)$ and $\left(x_{2}, 0\right)$, respectively. (b) The resultant electric field intensity landscape is highly asymmetric. (c) Force $F_{x 1}$ on the particle at $\left(x_{1}, 0\right)$. (d) Force $F_{x 2}$ on the particle at $\left(x_{2}, 0\right)$. The single-particle equilibrium positions $x_{1}=0$ and $x_{2}=480$ are marked with vertical and horizontal lines, respectively, in the force plots. 
shifted by less than $20 \mathrm{~nm}$. Moving the former does not affect the equilibrium position that significantly either. We can also consider the combined equilibrium of the two particles. This is the pair of locations when the restoring force on both particles vanish. It thus corresponds to the intersection of the white curves showing equilibria in Figs. 5(c) and 5(d). Surprisingly, the second particle is seen to be barely displaced from its original equilibrium location, whereas the first particle has shifted towards it by more than $100 \mathrm{~nm}$. The phase asymmetry in the trapping field has thus been clearly magnified in the two-particle response. The result is that at equilibrium, the two particles are much closer than the separation between intensity maxima in the unperturbed lattice. Having more nearby particles would modify the optical binding force and the equilibrium positions further.

\section{CONCLUSION}

We have proved theoretically that the three-beam optical lattice provides stable trap sites for all particles with threefold rotational symmetry and two flip symmetries irrespective of their specific electromagnetic response. Though the restoring force is isotropic for small displacements, the trap potential is asymmetric due to the phase dependence of the force. In turn, this strongly affects the optical binding force between the particles at adjacent trap sites. The presence of other particles in the lattice can thus modify the equilibrium positions and spring constants of the trap. This suggests that particle ensembles formed from the lattice could be significantly displaced from the expected positions, especially near the edges. This should be taken into consideration when performing experiments on optical matter. The modification of collective equilibria are expected to show interesting optomechanical behavior, which should be further investigated experimentally.

\section{ACKNOWLEDGMENT}

Funding from the European Research Council (Grant No. ERC-2015-AdG-695206 Nanofactory) is gratefully acknowledged.

\section{APPENDIX: RESTORING FORCE}

We can express the total electric and magnetic field as a field vector element $|\psi\rangle=|\mathbf{E}, \mathbf{H}\rangle$. Let us now define a vectorvalued operator $T$ acting on two field vector elements $\left|\psi^{1}\right\rangle$ and $\left|\psi^{2}\right\rangle$,

$$
\begin{aligned}
\left\langle\psi^{1}|T| \psi^{2}\right\rangle_{i}= & \frac{1}{2} \oint_{S}\left[\epsilon_{0} E_{i}^{1 *} E_{j}^{2}+\mu_{0} H_{i}^{1 *} H_{j}^{2}\right. \\
& \left.-\frac{1}{2} \delta_{i j} \sum_{k}\left(\epsilon_{0} E_{k}^{1 *} E_{k}^{2}+\mu_{0} H_{k}^{1 *} H_{k}^{2}\right)\right] n_{j} d S,
\end{aligned}
$$

where $\mathbf{E}^{m}$ and $\mathbf{H}^{m}$ are the electric and magnetic fields corresponding to the field vector element $\left|\psi^{m}\right\rangle$. The stress tensor integral for the force in Eqs. (1) and (2) reduces to applying the operator $T$ on the field vector element $|\psi\rangle$,

$$
\mathbf{F}=\operatorname{Re}\langle\psi|T| \psi\rangle
$$

It is easily seen from the definition that $T$ is conjugate linear in the first argument and linear in the second, as they are chosen to correspond to the first and second fields in each term of Eq. (2). If there are multiple illuminations which individually result in field vector elements $\left|\psi^{m}\right\rangle$, under the assumption that the response of the particle is linear, the field vector element as a result of the combination of all the illuminations is given by $|\psi\rangle=\sum_{m}\left|\psi^{m}\right\rangle$. The total force on the particle is then

$$
\begin{aligned}
\mathbf{F} & =\operatorname{Re}\left(\sum_{m}\left\langle\psi^{m}\right|\right)|T|\left(\sum_{n}\left|\psi^{n}\right\rangle\right) \\
& =\operatorname{Re} \sum_{m, n}\left\langle\psi^{m}|T| \psi^{n}\right\rangle .
\end{aligned}
$$

That is, the total force can be found by applying the operator on the fields pairwise. The sphere is illuminated by three waves. Let the field vector elements due to the waves propagating along $\hat{k}^{1}, \hat{k}^{2}$, and $\hat{k}^{3}$ when the sphere is at the origin be $\left|\psi^{1}\right\rangle,\left|\psi^{2}\right\rangle$, and $\left|\psi^{3}\right\rangle$, respectively. Defining the $120^{\circ}$ rotation operator $R$, we have from symmetry

$$
\left|\psi^{3}\right\rangle=R\left|\psi^{2}\right\rangle=R^{2}\left|\psi^{1}\right\rangle .
$$

We will use the following properties:

$$
\begin{aligned}
R^{3}|\psi\rangle & =|\psi\rangle, \\
|\psi\rangle+R|\psi\rangle+R^{2}|\psi\rangle & =0 \quad \text { (for in-plane vectors), }
\end{aligned}
$$

and

$$
\left(\left\langle\psi^{m}\right| R\right)|T|\left(R\left|\psi^{n}\right\rangle\right)=R\left\langle\psi^{m}|T| \psi^{n}\right\rangle .
$$

When the center of the sphere is displaced from the origin to $\mathbf{r}_{0}=\left(x_{0}, y_{0}\right)$, the three total fields due to the three beams get multiplied by different phase factors. The field vector element due to the $m$ th beam would now be

$$
\exp \left(i \frac{2 \pi}{\lambda} \hat{k}^{m} \cdot \mathbf{r}_{0}\right)\left|\psi^{m}\right\rangle=\exp \left(i \phi^{m}\right)\left|\psi^{m}\right\rangle .
$$

The total force on the sphere centered at $\mathbf{r}_{0}$ is hence

$$
\mathbf{F}\left(\mathbf{r}_{0}\right)=\operatorname{Re} \sum_{m, n} \exp \left[i\left(\phi^{n}-\phi^{m}\right)\right]\left\langle\psi^{m}|T| \psi^{n}\right\rangle .
$$

For compactness of notation, we will henceforth denote $\phi^{m}-$ $\phi^{n}$ by $\phi^{m n}$ and $\left\langle\psi^{m}|T| \psi^{n}\right\rangle$ by $T^{m n}$.

Considering only the terms with $m=n$,

$$
\begin{aligned}
\sum_{m} T^{m m} & =T^{11}+\left\langle\psi^{1}|R T R| \psi^{1}\right\rangle+\left\langle\psi^{1}\left|R^{2} T R^{2}\right| \psi^{1}\right\rangle \\
& =T^{11}+R T^{11}+R^{2} T^{11} \\
& =\left[1+R+R^{2}\right] T^{11} \\
& =0
\end{aligned}
$$

where we have used Eqs. (A4), (A5b), and (A5c). The terms with $m=n$ have canceled out. Note that $T^{m m}$ would be the force on the particle if it was illuminated by only the $m$ th beam. This first-order force has hence vanished due to symmetry and we are only left with the coupling terms $m \neq n$. Thus we 
obtain

$$
\begin{aligned}
\mathbf{F}\left(\mathbf{r}_{0}\right)= & \operatorname{Re}\left\{\exp \left[i \phi^{21}\right] T^{12}+\exp \left[i \phi^{32}\right] T^{23}+\exp \left[i \phi^{13}\right] T^{31}\right. \\
& \left.+\exp \left[i \phi^{12}\right] T^{21}+\exp \left[i \phi^{23}\right] T^{32}+\exp \left[i \phi^{31}\right] T^{13}\right\} \\
= & \operatorname{Re}\left\{\exp \left[i \phi^{21}\right] R^{2} T^{23}+\exp \left[i \phi^{32}\right] T^{23}+\exp \left[i \phi^{13}\right] R T^{23}\right. \\
& \left.+\exp \left[i \phi^{12}\right] R^{2} T^{32}+\exp \left[i \phi^{23}\right] T^{32}+\exp \left[i \phi^{31}\right] R T^{32}\right\} \\
= & \operatorname{Re}\left\{\left[\cos \phi^{21} R^{2}+\cos \phi^{32}+\cos \phi^{13} R\right]\left[T^{23}+T^{32}\right]\right. \\
& \left.+i\left[\sin \phi^{21} R^{2}+\sin \phi^{32}+\sin \phi^{13} R\right]\left[T^{23}-T^{32}\right]\right\}
\end{aligned}
$$

The total force can thus be expressed in terms of the 2-3 coupling alone (note that we could have done the same with 1-2 or
3-1 coupling as they are identical up to a rotation). To further simplify this, considering only the in-plane components, let

$$
T^{23}=a^{23} \hat{x}+b^{23} \hat{y},
$$

where $a^{23}$ and $b^{23}$ are complex numbers. If we rotate the coordinate system by $180^{\circ}$ about the $x$ axis, the identities of beams 2 and 3 are switched, and the direction of the $y$ axis is flipped as well, whereas the $x$ axis remains invariant. From this we can see that

$$
T^{32}=a^{23} \hat{x}-b^{23} \hat{y} .
$$

Setting $U^{23}=2 \operatorname{Re}\left(a^{23}\right)$ and $V^{23}=-2 \operatorname{Im}\left(b^{23}\right)$, we obtain Eq. (3).
[1] J. D. Jackson, Classical Electrodynamics, 3rd ed. (Wiley, New York, 1998).

[2] A. Ashkin, Acceleration and Trapping of Particles by Radiation Pressure, Phys. Rev. Lett. 24, 156 (1970).

[3] A. Ashkin, J. M. Dziedzic, J. E. Bjorkholm, and S. Chu, Observation of a single-beam gradient force optical trap for dielectric particles, Opt. Lett. 11, 288 (1986).

[4] J. Chen, J. Ng, Z. Lin, and C. T. Chan, Optical pulling force, Nat. Photon. 5, 531 (2011).

[5] M. Capitanio, R. Cicchi, and F. S. Pavone, Continuous and timeshared multiple optical tweezers for the study of single motor proteins, Opt. Laser. Eng. 45, 450 (2007).

[6] O. M. Maragò, P. H. Jones, P. G. Gucciardi, G. Volpe, and A. C. Ferrari, Optical trapping and manipulation of nanostructures, Nat. Nanotechnol. 8, 807 (2013).

[7] P. Bronkhorst, G. Streekstra, J. Grimbergen, E. Nijhof, J. Sixma, and G. Brakenhoff, A new method to study shape recovery of red blood cells using multiple optical trapping, Biophys. J. 69, 1666 (1995).

[8] D. G. Grier, A revolution in optical manipulation, Nature (London) 424, 810 (2003).

[9] K. C. Neuman and S. M. Block, Optical trapping, Rev. Sci. Instrum. 75, 2787 (2004).

[10] M. Righini, A. S. Zelenina, C. Girard, and R. Quidant, Parallel and selective trapping in a patterned plasmonic landscape, Nat. Phys. 3, 477 (2007).

[11] J. R. Moffitt, Y. R. Chemla, S. B. Smith, and C. Bustamante, Recent advances in optical tweezers, Annu. Rev. Biochem. 77, 205 (2008).

[12] L. Huang, S. J. Maerkl, and O. J. F. Martin, Integration of plasmonic trapping in a microfluidic environment, Opt. Express 17, 6018 (2009).

[13] R. Bowman, G. Gibson, and M. Padgett, Particle tracking stereomicroscopy in optical tweezers: Control of trap shape, Opt. Express 18, 11785 (2010).

[14] S. Lin, E. Schonbrun, and K. Crozier, Optical manipulation with planar silicon microring resonators, Nano Lett. 10, 2408 (2010).

[15] K. Ramser and D. Hanstorp, Optical manipulation for single-cell studies, J. Biophoton. 3, 187 (2010).

[16] K. Wang, E. Schonbrun, P. Steinvurzel, and K. B. Crozier, Scannable plasmonic trapping using a gold stripe, Nano Lett. 10, 3506 (2010).

[17] M. L. Juan, M. Righini, and R. Quidant, Plasmon nano-optical tweezers, Nat. Photon. 5, 349 (2011).
[18] Y. Pang and R. Gordon, Optical trapping of a single protein, Nano Lett. 12, 402 (2012).

[19] B. J. Roxworthy, K. D. Ko, A. Kumar, K. H. Fung, E. K. C. Chow, G. L. Liu, N. X. Fang, and K. C. Toussaint, Application of plasmonic bowtie nanoantenna arrays for optical trapping, stacking, and sorting, Nano Lett. 12, 796 (2012).

[20] S. Lin and K. B. Crozier, Trapping-assisted sensing of particles and proteins using on-chip optical microcavities, ACS Nano 7, 1725 (2013).

[21] T. V. Raziman, R. J. Wolke, and O. J. F. Martin, Optical forces in nanoplasmonic systems: How do they work, what can they be useful for? Faraday Discuss. 178, 421 (2015).

[22] P. Y. Chiou, A. T. Ohta, and M. C. Wu, Massively parallel manipulation of single cells and microparticles using optical images, Nature (London) 436, 370 (2005).

[23] T. Čižmár, L. C. Dávila Romero, K. Dholakia, and D. L. Andrews, Multiple optical trapping and binding: New routes to self-assembly, J. Phys. B 43, 102001 (2010).

[24] V. Garcés-Chávez, D. McGloin, H. Melville, W. Sibbett, and K. Dholakia, Simultaneous micromanipulation in multiple planes using a self-reconstructing light beam, Nature (London) 419, 145 (2002).

[25] K. Dholakia and W. Lee, Optical trapping takes shape: The use of structured light fields, Adv. At. Mol. Opt. Phys. 56, 261 (2008).

[26] E. R. Dufresne and D. G. Grier, Optical tweezer arrays and optical substrates created with diffractive optics, Rev. Sci. Instrum. 69, 1974 (1998).

[27] E. R. Dufresne, G. C. Spalding, M. T. Dearing, S. A. Sheets, and D. G. Grier, Computer-generated holographic optical tweezer arrays, Rev. Sci. Instrum. 72, 1810 (2001).

[28] J. Liesener, M. Reicherter, T. Haist, and H. Tiziani, Multifunctional optical tweezers using computer-generated holograms, Opt. Commun. 185, 77 (2000).

[29] M. Woerdemann, C. Alpmann, M. Esseling, and C. Denz, Advanced optical trapping by complex beam shaping, Laser Photon. Rev. 7, 839 (2013).

[30] D. Preece, R. Bowman, A. Linnenberger, G. Gibson, S. Serati, and M. Padgett, Increasing trap stiffness with position clamping in holographic optical tweezers, Opt. Express 17, 22718 (2009).

[31] F. Merenda, J. Rohner, J.-M. Fournier, and R.-P. Salathé, Miniaturized high-NA focusing-mirror multiple optical tweezers, Opt. Express 15, 6075 (2007).

[32] J.-M. R. Fournier, M. M. Burns, and J. A. Golovchenko, Writing diffractive structures by optical trapping, in Practical 
Holography IX, edited by S. A. Benton, SPIE Proc. Vol. 2406 (SPIE Bellingham, 1995), p. 101.

[33] M. MacDonald, S. Neale, L. Paterson, A. Richies, K. Dholakia, and G. Spalding, Cell cytometry with a light touch: Sorting microscopic matter with an optical lattice, J. Biol. Regul. Homeostatic Agents 18, 200 (2004).

[34] A. Casaburi, G. Pesce, P.Zemánek, and A. Sasso, Two- and threebeam interferometric optical tweezers, Opt. Commun. 251, 393 (2005).

[35] E. Schonbrun, R. Piestun, P. Jordan, J. Cooper, K. D. Wulff, J. Courtial, and M. Padgett, 3D interferometric optical tweezers using a single spatial light modulator, Opt. Express 13, 3777 (2005).

[36] M. M. Burns, J.-M. Fournier, and J. A. Golovchenko, Optical matter: Crystallization and binding in intense optical fields, Science 249, 749 (1990).

[37] J.-M. R. Fournier, G. Boer, G. Delacretaz, P. M. Jacquot, J. Rohner, and R. P. Salathe, in Optical Trapping and Optical Micromanipulation, edited by K. Dholakia and G. C. Spalding, SPIE Proc. Vol. 5514 (SPIE, Bellingham, 2004), p. 309.

[38] B. N. Slama-Eliau and G. Raithel, Three-dimensional arrays of submicron particles generated by a four-beam optical lattice, Phys. Rev. E 83, 051406 (2011).

[39] A. Dereux, C. Girard, O. J. F. Martin, and M. Devel, Optical binding in scanning probe microscopy, Europhys. Lett. 26, 37 (1994).

[40] C. Girard, A. Dereux, and O. J. F. Martin, Theoretical analysis of light-inductive forces in scanning probe microscopy, Phys. Rev. B 49, 13872 (1994).

[41] M. Guillon, O. Moine, and B. Stout, Longitudinal Optical Binding of High Optical Contrast Microdroplets in Air, Phys. Rev. Lett. 96, 143902 (2006).

[42] M. M. Burns, J.-M. Fournier, and J. A. Golovchenko, Optical Binding, Phys. Rev. Lett. 63, 1233 (1989).

[43] K. Dholakia and P. Zemánek, Colloquium, Rev. Mod. Phys. 82, 1767 (2010).

[44] S. A. Tatarkova, A. E. Carruthers, and K. Dholakia, OneDimensional Optically Bound Arrays of Microscopic Particles, Phys. Rev. Lett. 89, 283901 (2002).

[45] H. Yoshikawa, T. Matsui, and H. Masuhara, Reversible assembly of gold nanoparticles confined in an optical microcage, Phys. Rev. E 70, 061406 (2004).
[46] V. Garcés-Chávez, K. Dholakia, and G. C. Spalding, Extendedarea optically induced organization of microparticles on a surface, Appl. Phys. Lett. 86, 031106 (2005).

[47] J. Ng, Z. F. Lin, C. T. Chan, and P. Sheng, Photonic clusters formed by dielectric microspheres: Numerical simulations, Phys. Rev. B 72, 085130 (2005).

[48] T. M. Grzegorczyk, B. A. Kemp, and J. A. Kong, Stable Optical Trapping Based on Optical Binding Forces, Phys. Rev. Lett. 96, 113903 (2006).

[49] B. A. Grzybowski, C. E. Wilmer, J. Kim, K. P. Browne, and K. J. M. Bishop, Self-assembly: From crystals to cells, Soft Matter 5, 1110 (2009).

[50] T. M. Grzegorczyk, J. Rohner, and J.-M. Fournier, Optical Mirror from Laser-Trapped Mesoscopic Particles, Phys. Rev. Lett. 112, 023902 (2014).

[51] A. M. Kern and O. J. F. Martin, Surface integral formulation for 3D simulations of plasmonic and high permittivity nanostructures, J. Opt. Soc. Am. A 26, 732 (2009).

[52] A. Ji, T. V. Raziman, J. Butet, R. P. Sharma, and O. J. F. Martin, Optical forces and torques on realistic plasmonic nanostructures: A surface integral approach, Opt. Lett. 39, 4699 (2014).

[53] T. V. Raziman, W. R. C. Somerville, O. J. F. Martin, and E. C. Le Ru, Accuracy of surface integral equation matrix elements in plasmonic calculations, J. Opt. Soc. Am. B 32, 485 (2015).

[54] P. B. Johnson and R. W. Christy, Optical constants of the noble metals, Phys. Rev. B 6, 4370 (1972).

[55] C. Oubre and P. Nordlander, Optical properties of metallodielectric nanostructures calculated using the finite difference time domain method, J. Phys. Chem. B 108, 17740 (2004).

[56] See Supplemental Material at http://link.aps.org/supplemental/ 10.1103/PhysRevA.98.023420 for a video of the temporal evolution of the electric field showing the phase difference between the different maxima.

[57] J. P. Gordon, Radiation forces and momenta in dielectric media, Phys. Rev. A 8, 14 (1973).

[58] L. Huang and O. J. F. Martin, Reversal of the optical force in a plasmonic trap, Opt. Lett. 33, 3001 (2008).

[59] T. M. Grzegorczyk, B. A. Kemp, and J. A. Kong, Trapping and binding of an arbitrary number of cylindrical particles in an in-plane electromagnetic field, J. Opt. Soc. Am. A 23, 2324 (2006). 